\title{
THE DOUBLE CENTRALIZER PROPERTY IS CATEGORICAL ${ }^{1}$
}

\author{
R. S. CUNNINGHAM AND E. A. RUTTER, JR.
}

The purpose of this note is to prove the result stated in the title. This answers a question raised by E. A. Walker at the summer symposium on ring theory at Appalachian State University in August, 1969 , as to whether being QF-1 is categorical. This also seems timely since many papers have appeared recently studying the double centralizer property for modules, for instance [2], [4], and [6] .

Let $R$ and $S$ be associative rings with identity such that the categories $R \subset M$ and $s \subset M$ of left $R$-modules and left $S$-modules respectively are equivalent. Then by [7, Theorem 3.5] or [1, Theorem 3.4, p. 62] there is a right $R$-progenerator $P_{R}$ with $S \simeq \operatorname{End}_{R}\left(P_{R}\right)$ such that the functor $F={ }_{s} P_{R} \otimes_{R}(-):{ }_{R} \mathcal{M} \rightarrow \mathrm{s} \wedge M$ gives the equivalence; we say $R$ and $S$ are Morita equivalent via ${ }_{S} P_{R}$.

Let $M$ be a left $R$-module and let $C=\operatorname{End}_{R}\left({ }_{R} M\right)$. Then the map $C \rightarrow \operatorname{End}_{S}\left({ }_{s} P \otimes_{R} M\right)$ via $f \rightarrow 1_{P} \otimes f$ is a unital ring isomorphism so we identify $C$ with $\operatorname{End}_{S}\left(P \otimes_{R} M\right)$. Let $D=\operatorname{End}_{C}\left(M_{C}\right)$ and $E=$ $\operatorname{End}_{C}\left(P \otimes_{R} M_{C}\right)$ be the double centralizers of ${ }_{R} M$ and ${ }_{S} P \otimes_{R} M=$ ${ }_{s} F(M)$ respectively; note that we write homomorphisms opposite scalars. We say that $M$ has the double centralizer property (DCP) if the natural map $\eta: R \rightarrow \operatorname{End}_{C}\left(M_{C}\right)$ via $\eta(r)(m)=r m$ is onto. Equivalently, if $\operatorname{Ann}_{R}(M)=\{r \in R \mid r m=0$ for all $m \in M\}, M$ has the DCP if the natural map $R / \operatorname{Ann}_{R}(M) \rightarrow \operatorname{End}_{C}(M)$ is an isomorphism.

Finally, we say that two modules $N$ and $N^{\prime}$ are similar if each is isomorphic to a direct summand of a finite direct sum of copies of the other and in this case we write $N \sim N^{\prime}$.

Theonem. Let $R$ and $S$ be Morita equivalent via the module ${ }_{\mathrm{S}} \boldsymbol{P}_{\mathrm{R}}$. If a left R-module ${ }_{\mathrm{R}} M$ has the double centralizer property, so does $_{S} F(M)={ }_{S} P \otimes_{R} M$.

Proof. Since $P_{R}$ is a progenerator, $P_{R} \sim R_{R}$, so $P \otimes_{R} M \sim R \otimes_{R} M$ $\cong M$ as abelian groups, and the action of $C$ on $P \otimes_{R} M$ makes

Received by the editors November 23, 1970 and, in revised form, March 19, 1971.

AMS 1970 subject classifications. Primary 16A36, 16A64.

${ }^{1} \mathrm{~K}$. R. Fuller tells us this result also appears in an unpublished paper by Morita and Tachikawa. We feel our proof is worth recording since it was obtained independently and no proof is available in print. 
$P \otimes_{R} M \sim M$ as right $C$-modules. Thus by [5, Theorem 1.5], $D$ and $E$ are Morita equivalent via the module ${ }_{E} \operatorname{Hom}_{C}\left(M, P \otimes_{R} M\right)_{D}$. Our proof of the theorem will be in two parts.

(a) If ${ }_{R} M$ is faithful and has the DCP we may identify $R$ and $D$. Then the Morita equivalences of $S$ and $R$ and of $R$ and $E$ give a Morita equivalence of $S$ and $E$ via the module ${ }_{E} \operatorname{Hom}_{C}\left(M, P \otimes_{R} M\right)$ $\otimes_{R} P_{S}{ }^{*}$ where $P^{*}=\operatorname{Hom}_{R}(P, R)$. Applying the Hom-Tensor identities [3, VI, Proposition 5.2] and [3, II, Proposition 5.2], recalling that $P_{R}$ is a progenerator, we have

$$
\begin{aligned}
\operatorname{Hom}_{C}\left(M, P \otimes_{R} M\right) \otimes_{R} P^{*} & \simeq \operatorname{Hom}_{C}\left(\operatorname{Hom}_{R}\left(P^{*}, M\right), P \otimes_{R} M\right) \\
& \simeq \operatorname{Hom}_{C}\left(P \otimes_{R} M, P \otimes_{R} M\right) \simeq E
\end{aligned}
$$

as left $E$-right $S$-bimodules. Thus ${ }_{E} E_{S}$ gives a Morita equivalence of $E$ and $S$ and we conclude by [1, Theorem 3.5, p. 65] that $S \simeq$ $\operatorname{End}_{E}(E) \simeq E$. Since this isomorphism is the canonical mapping and ${ }_{S} F(M)$ is faithful [1, Theorem 3.5, p. 65], ${ }_{S} P \otimes_{R} M={ }_{s} F(M)$ has the DCP.

(b) If ${ }_{R} M$ is not faithful, let $A=\operatorname{Ann}_{R}(M)$. Then the ideal $A^{\prime}$ of $S$ corresponding to $A$ under the lattice isomorphism of ideals of $R$ and $S$ is isomorphic to $\operatorname{Hom}_{R}(P, P A)$ and is the annihilator in $S$ of ${ }_{S} P \otimes_{R} M$ [1, Theorem 3.5, p. 65]. Let $R^{\prime}=R / A$ and $S^{\prime}=S / A^{\prime}$. Clearly $P / P A$ is a right $R^{\prime}$-progenerator. Since $P_{R}$ is projective, the natural ring homomorphism from $\operatorname{End}_{R}(P)$ to $\operatorname{End}_{R^{\prime}}(P / P A)$ via $f \rightarrow \bar{f}$ where $\bar{f}(p+P A)=f(p)+P A$ is surjective with kernel $\operatorname{Hom}_{R}(P, P A)$. It follows as noted above that $S^{\prime} \simeq \operatorname{End}_{R^{\prime}}(P / P A)$ so that $R^{\prime}$ and $S^{\prime}$ are Morita equivalent via ${ }_{S^{\prime}} P / P A_{R^{\prime}}$. Since $M$ is faithful over $R^{\prime}$, we may apply the first part of the proof to conclude that ${ }_{S} P \otimes_{R} M$ has the DCP provided we show that ${ }_{s} P \otimes_{R} M \simeq{ }_{s} P / P A \otimes_{R^{\prime}} M$. But $0 \rightarrow P A \rightarrow P \rightarrow P / P A \rightarrow 0$ is an exact sequence of left $S$-right $R$ bimodules, so tensoring with ${ }_{R} M$ we have the exact sequence of left $S$-modules

$$
P A \otimes_{R} M \rightarrow P \otimes_{R} M \rightarrow P / P A \otimes_{R} M \rightarrow 0 .
$$

Since the image of $P A \otimes_{R} M$ in $P \otimes_{R} M$ is zero, this shows $P \otimes_{R} M$ $\simeq P / P A \otimes_{R} M \simeq P / P A \otimes_{R^{\prime}} M$ as left $S$ - modules.

A ring $R$ is called left $Q F-1$ if every faithful left $R$-module has the DCP, while $R$ is called balanced if every left $R$-module has the DCP. See [2] for further background on these types of rings.

Corollary. Let $R$ and $\mathrm{S}$ be Morita equivalent.

(a) If $R$ is left $Q F-1$, then $S$ is left $Q F-1$.

(b) If $R$ is balanced, then $S$ is balanced. 
In particular, if $S$ is a full $n \times n$ matric ring over $R$, then $S$ is left $Q F-1$ or balanced if and only if $R$ is.

\section{REFERENCES}

1. H. Bass, Algebraic K-theory, Benjamin, New York, 1968. MR 40 \#2736.

2. V. P. Camillo, Balanced rings and a problem of Thrall, Trans. Amer. Math. Soc. 149 (1970), 143-153. MR 41 \#5417.

3. H. Cartan and S. Eilenberg, Homological algebra, Princeton Univ. Press, Princeton, N. J., 1956. MR 17, 1040.

4. K. R. Fuller, Generalized uniserial rings and their Kupisch series, Math. Z. 106 (1968), 248-260. MR 38 \#1118.

5. K. Hirata, Some types of separable extensions of rings, Nagoya Math. J. 33 (1968), 107-115. MR 38 \#4524.

6. J. P. Jans, Note on QF-1 algebras, Proc. Amer. Math. Soc. 20 (1969), 225-228. MR 38 \#2169.

7. K. Morita, Duality for modules and its applications to the theory of rings with minimum condition, Sci. Rep. Tokyo Kyoiku Daigaku Sect. A 6 (1958), 83-142. MR 20 \#3183.

The University of Kansas, Lawrence, Kansas 66044 
